EPJ Web of Conferences 47, 03002 (2013)

DOI: $10.1051 /$ epjconf/20134703002

(C) Owned by the authors, published by EDP Sciences, 2013

\title{
The Pan-STARRS1 Planet Survey: Overview and first results
}

\author{
J. Koppenhoefer ${ }^{1,2, a}$, Th. Henning ${ }^{3}$, R.P. Saglia ${ }^{1,2}$, C. Obermeier ${ }^{2,3}$, \\ S. Kretschmann ${ }^{2}$ and N. Nikolov 3,4 \\ ${ }^{1}$ University Observatory Munich, Scheinerstrasse 1, 81679 München, Germany \\ ${ }^{2}$ Max-Planck-Institute for Extraterrestrial Physics, Giessenbachstrasse, 85748 Garching, \\ Germany \\ ${ }^{3}$ Max-Planck-Institute for Astronomy, Königstuhl 17, 69117 Heidelberg, Germany \\ ${ }^{4}$ University of Exeter, Stocker Road, Exeter EX4 4QL, UK
}

\begin{abstract}
The Pan-STARRS1 Planet Survey (Pan-Planets) is a search for transiting extra-solar planets in the Galactic disk with a focus on planets around M-dwarfs and White dwarfs. The large field of view of the Pan-STARRS1 camera enables us to monitor $\sim 50000$ M-dwarfs and $\sim 5000$ White dwarfs brighter than $\mathrm{i}_{P 1}=18$ in seven fields. We give a description of the science goals and observating strategy and present some early results from the first two observing campaigns conducted in 2010 and 2011.
\end{abstract}

\section{INTRODUCTION}

Up to now almost 300 transiting planets in extra-solar systems have been detected $^{1}$ covering a wide range of physical parameters such as size, density or orbital period. Most ongoing ground based transit surveys follow an all-sky approach targeting bright stars using small aperture telescopes. The number of M-dwarfs and White dwarfs observed in these surveys is low, due to the bright magnitude limit of the target populations.

The space missions Kepler [1] and CoRoT [2] marked a new age of transit detection. From space, an unprecedented precision allows to study the population of extra-solar planets down to Earth-radii with great statistics. However, the sample of M-dwarfs observed in these missions is in the order of a few thousand only, making a detection of a large sample of planets around M-dwarfs unlikely.

Since several years the MEarth project [3] is monitoring a few thousand bright M-dwarfs with a network of small telescopes. The WFCAM Transit Survey (WTS, Kovacs et al. 2013, submitted) is a deep J-band survey for planets around cool stars with the UKIRT on Hawaii. The Palomar Transient Factory [4] is observing on the order of $100000 \mathrm{M}$-dwarfs to search for transiting planets. This is the largest sample of M-dwarfs monitored in a transit survey up to now, however, so far no detection has been reported.

Planets orbiting M-dwarfs and White dwarfes are of particular interest since their habitable zone is much closer to the host star compared to planets around other stellar types. Up to now, no planet has been discovered orbiting a White dwarf. Any detection would have a great impact on the understanding of the evolution of planetary systems beyond the main-sequence phase of the host star. Around M-dwarfs, several planets have been detected. However, the fraction of low-mass stars with Jupitersized companions is not known very accurately. Kovacs et al. (2013, submitted) presented an upper limit of 2\% for M0-M1 and 3\% for M2-M4 for planets with period below 10 days as found in the WTS

\footnotetext{
ae-mail: koppenh@mpe.mpg.de

1 www .exoplanet.eu
}

This is an Open Access article distributed under the terms of the Creative Commons Attribution License 2.0, which permits unrestricted use, distribution, and reproduction in any medium, provided the original work is properly cited. 


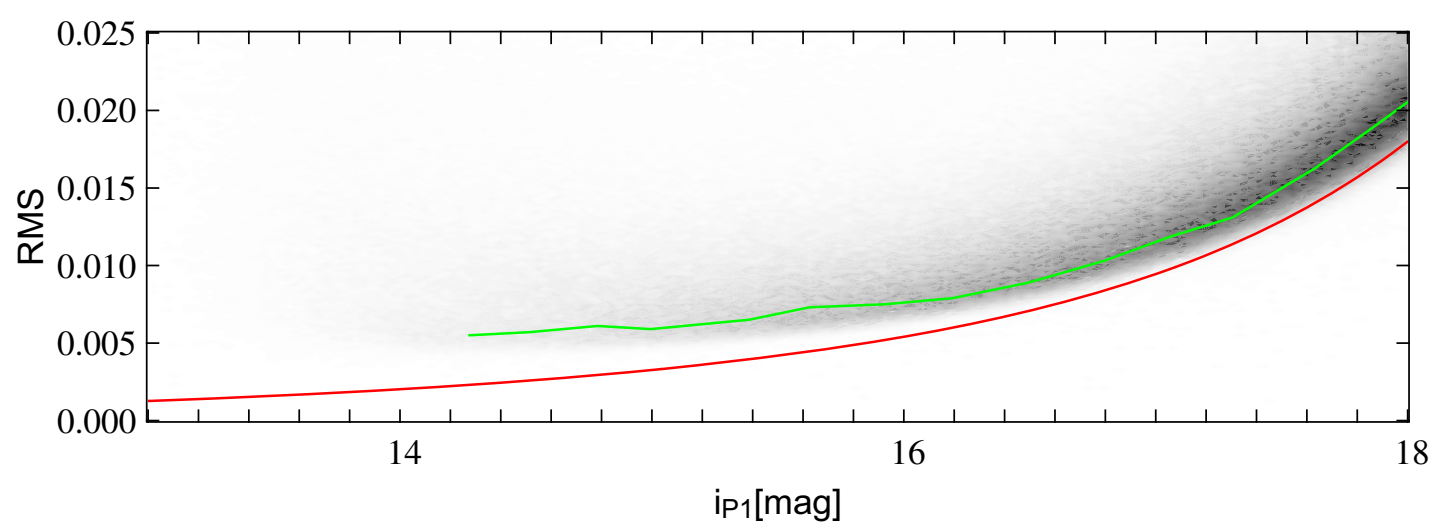

Figure 1. Photometric precision achieved in Pan-Planets as a function of $i_{P 1}$-band magnitude as measured on one field with two years of data (i.e. 2010 and 2011). We iteratively clipped 5- $\sigma$ outliers and calculated the RMS of all remaining measurements with respect to the average flux. The red curve shows the theoretical RMS based on photon and readout noise for typical observing conditions. The green curve marks the mode of the RMS distribution in magnitude bins.

in the analysis of the first of four fields down to magnitudes of $J=16$. With Pan-Planets we aim at the detection of these rare types of objects. The key to success will be the large area in combination with a high photometric precision down to faint magnitudes.

\section{PAN-PLANETS}

Pan-Planets is one of the key projects of the Pan-STARRS1 (PS1) science consortium. The survey utilizes the PS1 telescope [5] which is operated at Haleakala Observatory. In the period from May 2010 to October 2012 we obtained 170 hours of observations (about $4 \%$ of the available observing time).

The survey strategy has been optimized using Monte-Carlo simulations [6]. Seven slightly overlapping fields of 7 sq. deg. each are observed for approximately 1 hour per night at low Galactic latitude $\left(b \sim 3-7^{\circ}\right)$ in the Cygnus region of the Milky Way. In the first season in 2010 only three of the seven fields have been observed. With an exposure and read-out time of $30 \mathrm{~s}$ and $12 \mathrm{~s}$ respectively, the observational cadence is roughly $2 \mathrm{~min}$ in 2010 and $5 \mathrm{~min}$ in 2011 and 2012.

The Pan-Planets data reduction pipeline is based on the MDia package [7] which is an implementation of the difference imaging algorithm proposed by Alard \& Lupton [8] that has been integrated into the Astro-WISE environment [9]. The procedure is making use of a deep reference stack of the images with the narrowest PSF. This reference image is broadened by convolution to match the PSF of the individual images and subsequently subtracted. The light curves are created by performing PSF-photometry on the difference images to measure the differential fluxes and then adding the flux measured in the reference image to each data point. For further details we refer to [7].

The light curve analysis includes removal of systematics with the sysrem algorithm [10] and a scaling of the error bars that is depending on the magnitude of each object. We detect periodically eclipsing systems with a box-fitting least squares algorithm. Our implementation which is based on [11] includes several modifications to improve the selection of high quality candidates [12].

\section{RESULTS}

The total number of stars in the magnitude range $i_{P 1}=13.5-18$ mag in the 40 sq. deg. Pan-Planets area is 1.5 Mio. Based on SED-fitting and proper motion we selected $\sim 50000 \mathrm{M}$-dwarf and $\sim 5000$ White 


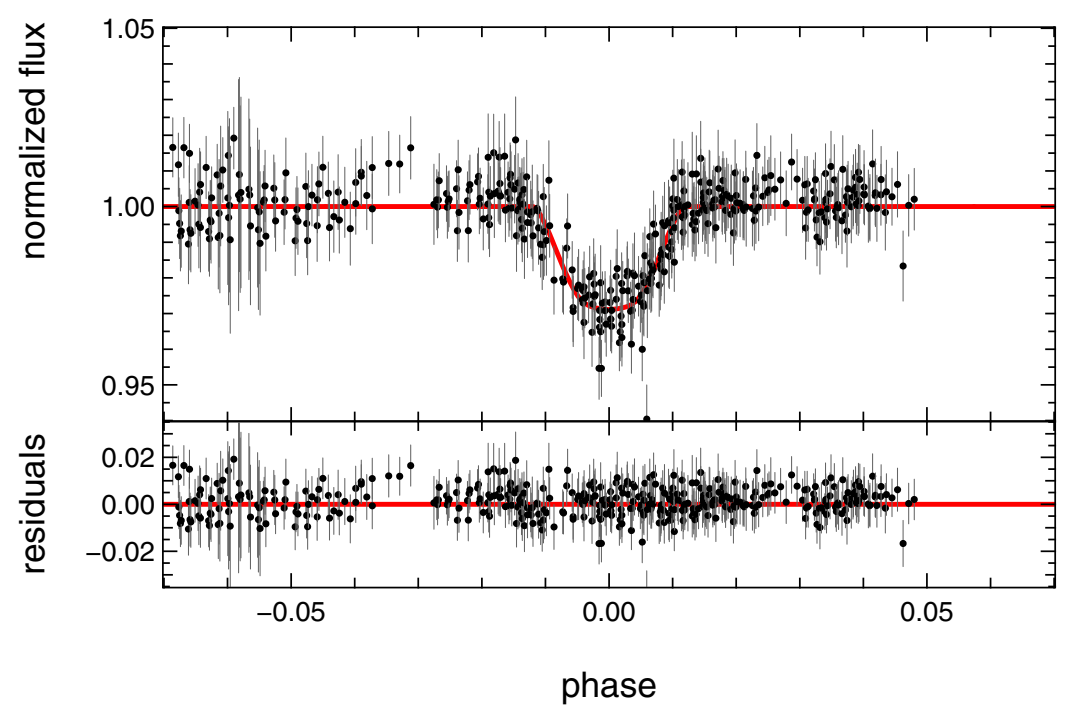

Figure 2. Pan-Planets candidate found in the sample of comparably bright FGK-stars. The period is 4.2 days. With a depth of $\sim 3 \%$ the planet radius is $1.7 \mathrm{R}_{\text {Jup }}$ assuming a late G-type host. The red line shows the best fitting analytic model according to the formulae of [13].

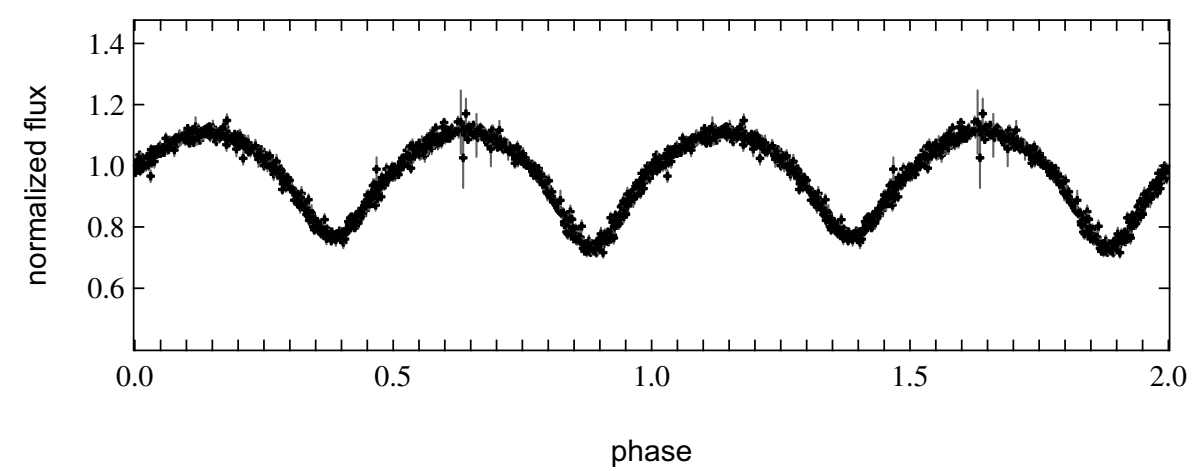

Figure 3. Ultra-short period eclipsing binary found in the analysis of the 2010 and 2011 data. With a period of $\sim 0.217$ days this $\mathrm{i}_{P 1}=18.2$ mag object belongs to a rare species of systems close to the sharp cut-off period found in previous studies [14].

dwarf candidates. Until now we processed the data of the first two campaigns in 2010 and 2012 for one and a half of the seven fields. Figure 1 shows the photometric precision we achieved. The red line shows the expected precision based on photon and readout noise statistics. At the bright end we reach a level of $\sim 5$ mmag which is slightly higher than the theoretical value.

We found four planet candidates around M-dwarfs which are currently being followed-up with lowresolution spectroscopy at the Hobby-Eberly Telescope. Photometric observations with the Calar Alto $1.23 \mathrm{~m}$ telescope centered on predicted transit times will allow us to derive more precise period estimates for our candidates and provide light curves with a better time resolution which enable us to remove false detections. We also found four candidates around bright stars of earlier spectral type. Figure 2 shows a $1.7 \mathrm{R}_{\text {Jup }}$ candidate orbiting an $\mathrm{i}_{P 1}=15.1$ mag G-type star in 4.05 days.

In addition we are following-up a few planet candidates detected around White dwarfs. These objects show extremely short transits (in the order of a few minutes only) and therefore have only few points 
in transit. Including the data from the third campaign in 2012 in the analysis will clarify if the detected periodicity proves to be consistent or not.

Figure 3 shows an extremely close eclipsing binary system with a period of 0.217 days only. So far we found eight of such systems and expect to find many more in the full Pan-Planets data set.

\section{References}

[1] M. Auvergne, P. Bodin, L. Boisnard, J.T. Buey, S. Chaintreuil, G. Epstein, M. Jouret, T. Lam-Trong, P. Levacher, A. Magnan et al., A\&A 506, 411 (2009)

[2] W.J. Borucki, D. Koch, G. Basri, N. Batalha, T. Brown, D. Caldwell, J. Caldwell, J. ChristensenDalsgaard, W.D. Cochran, E. DeVore et al., Science 327, 977 (2010)

[3] J. Irwin, D. Charbonneau, P. Nutzman, E. Falco (2009), Vol. 253, pp. 37-43

[4] N.M. Law, A.L. Kraus, R.R. Street, T. Lister, A. Shporer, L.A. Hillenbrand, Palomar Transient Factory Collaboration (2011), Vol. 448, p. 1367

[5] N. Kaiser, W. Burgett, K. Chambers, L. Denneau, J. Heasley, R. Jedicke, E. Magnier, J. Morgan, P. Onaka, J. Tonry (2010), Vol. 7733

[6] J. Koppenhoefer, C. Afonso, R.P. Saglia, T. Henning, A\&A 494, 707 (2009)

[7] J. Koppenhoefer, R.P. Saglia, A. Riffeser, Exp. Astr. p. 141 (2011)

[8] C. Alard, R.H. Lupton, ApJ 503, 325 (1998)

[9] E.A. Valentijn, J.P. McFarland, J. Snigula, K.G. Begeman, D.R. Boxhoorn, R. Rengelink, E. Helmich, P. Heraudeau, G. Verdoes Kleijn, R. Vermeij et al. (2007), Vol. 376, p. 491

[10] O. Tamuz, T. Mazeh, S. Zucker, MNRAS 356, 1466 (2005)

[11] G. Kovács, S. Zucker, T. Mazeh, A\&A 391, 369 (2002)

[12] J. Zendejas, J. Koppenhoefer, R.P. Saglia, et al., in preperation (2013)

[13] K. Mandel, E. Agol, ApJ 580, L171 (2002)

[14] S.V. Nefs, J.L. Birkby, I.A.G. Snellen, S.T. Hodgkin, D.J. Pinfield, B. Sipőcz, G. Kovacs, D. Mislis, R.P. Saglia, J. Koppenhoefer et al., MNRAS 425, 950 (2012) 